\title{
Incidence and Impact of Adverse Effects to Antibiotics in Hospitalized Adults with Pneumonia
}

\author{
Robert Y. Lin, MD ${ }^{1,2}$ \\ Farzana Nuruzzaman, вА ${ }^{2}$ \\ Shaili N. Shah, вA $^{2}$ \\ ${ }^{1}$ Department of Medicine, St. Vincent's Hospital- \\ Manhattan-Saint Vincent Catholic Medical Cen- \\ ters (SVCMC), New York, New York. \\ ${ }^{2}$ New York Medical College, School of Medicine, \\ Valhalla, New York.
}

BACKGROUND: This study sought to define the incidence, economic impact, and nature of adverse drug effects (ADEs) related to antibiotics in pneumonia hospitalizations in the US.

METHODS: Adult pneumonia hospitalizations were tabulated in statewide (New York) and national databases, respectively, from 2000 through 2005. The incidences of antibiotic related ADEs were determined by identifying antibiotic specific e-codes (external cause of injury codes). The modeled effect of the presence of antibiotic ADEs on length of stay (LOS) and total charges were also calculated. ADEs due to specific antibiotic classes, and the presence of certain cutaneous allergic and gastro-intestinal manifestations commonly attributable to ADEs, were tabulated.

RESULTS: ADEs related to antibiotics were reported in a small but consistent proportion $(0.45-0.6 \%)$ of pneumonia hospitalizations in both cohorts. The most common identifiable antibiotics class associated with ADEs was the cephalosporins followed by penicillins and quinolones. Over $60 \%$ of the ADEs were associated with reported dermal/allergic and gastro-intestinal manifestations. Multivariate analysis adjusting for co-morbid conditions and demographic factors showed that the presence of an antibiotic adverse drug effect was a significant independent predictor of greater LOS and higher total hospital charges.

CONCLUSIONS: ADEs related to antibiotics can be identified by analyzing administrative hospitalization databases. For pneumonia, a common hospitalization diagnosis, there is a defined calculable impact and incidence of antibiotic associated adverse effects. This should be considered in planning hospitalization resource allocation and in developing equitable hospitalization reimbursements. Identifying the nature of antibiotic associated adverse effects may facilitate the development of stratagies for reducing these adverse effects. Journal of Hospital Medicine 2009;4:E7-E15. () 2009 Society of Hospital Medicine.

KEYWORDS: adverse effects, antibiotics, hospitalization, pneumonia.

A dverse drug events-defined as an injury resulting from medical intervention related to a drug ${ }^{1}$ - significantly contribute to health care expenditures. Over 770,000 people are injured or die every year in hospitals from adverse drug events, and national hospital expenses to treat patients who have suffered adverse drug events during hospitalization have been estimated to be between $\$ 1.56$ and $\$ 4.2$ billion annually. ${ }^{2}$ In a meta-analysis of prospective studies, researchers found that adverse drug reactions, one important form of adverse drug events, may rank as the fourth to sixth leading cause of death in the United States, with more than 100,000 deaths per year. ${ }^{3}$ Understanding the factors associated with these adverse events may help in the development of prevention strategies, with resulting improving health care quality and lowering health care costs. 
Among hospitalized patients, antibacterial adverse effects may account for approximately $25 \%$ of adverse drug reactions. ${ }^{1,4}$ While the economic impact has been studied for overall adverse drug events in hospitalized patients in the 1990s, more recent detailed studies for the impact of antibiotic-related adverse drug effects have not been published. As hospitalized patients with the primary diagnosis of pneumonia are invariably treated with antibiotics, and since pneumonia is the third leading cause for hospitalization in the United States, ${ }^{5}$ hospitalization databases that document pneumonia hospitalizations as well as adverse effects from antibiotics, using specific International Classification of Diseases, Ninth Revision (ICD-9) clinical modification codes, constitute a unique and rich resource for quantifying and analyzing the incidence and impact of antibiotic-associated adverse drug effects.

The purpose of this study was to describe the incidence and clinical manifestations of adverse drug effects in pneumonia hospitalizations in recent years, and to determine the types of patients and comorbidities, which are most commonly associated with adverse drug effects. The term "adverse drug effect" refers more to known side effects of medications, whereas adverse drug events and adverse drug reactions refer to an injury or a noxious, unintended, and undesired effect resulting from administration of a drug. ${ }^{6}$ As this study utilized medical coding for data abstraction, the broader classifications of adverse drug events or reactions could not be examined and instead the outcome of "adverse drug effect" was utilized.

\section{METHODS}

\section{Data Sources}

The Statewide Planning and Research Cooperative System (SPARCS) database was accessed as previously described. ${ }^{7,8}$ There is mandatory reporting to this database for all New York State acute care hospitalizations. Each deidentified SPARCS admission record contains more than 100 data fields ${ }^{9}$ that consist of demographic, clinical, and financial information. These fields include principal and nonprincipal diagnostic fields, procedure codes, race, age, gender, and ethnicity information, hospital characteristics, expected reimbursement, total charges, length of stay (LOS), admission status, and disposition status. Both ICD-9 and Com- mon Procedural Terminology (CPT) codes are input for each admission.

The Nationwide Inpatient Sample (NIS) of the Healthcare Cost and Utilization Project (HCUP), contains annual hospital discharge data from a sample of US hospitals. ${ }^{10}$ Each NIS patient record includes data fields consisting of demographic, clinical, and financial information. These fields include primary and secondary diagnostic fields, procedure codes, age, gender, race, total charges, length of stay, payer codes, hospital characteristics, and disposition status. Twenty percent random subsamples from each year's sample were employed to perform the analyses. HCUP databases include appropriately-scaled discharge weights to generate national estimates of hospitalizations and total charges from the NIS. These weights allow comparison of incidence rates and charges across years despite a varying number of states and hospitals included in the database each year.

This study was given an exemption from institutional research board approval by the SVCMC Integrated Scientific and Ethical Review Board.

\section{Identification of Pneumonia Admissions}

To achieve more uniformity in the cohorts, it was decided to select only hospitalizations with the most common ICD-9 and diagnosis-related group (DRG) codes for pneumonia for more recent years. ICD-9 and DRG codes have been used to identify pneumonia patients in administrative data, and cases selected in this manner show excellent specificity when compared to a reference standard. ${ }^{11}$ Hospitalization discharges from both databases from 2000 through 2005 were selected with the criterion of having the principal diagnosis of ICD9 code 486 (pneumonia, organism unspecified) and the DRG of 89 (simple pneumonia and pleurisy, age $>17$ years, with complication and comorbidity), thus targeting community acquired pneumonia. These hospitalizations formed the 2 cohorts of pneumonia hospitalizations. Preliminary analyses showed that hospitalizations identified by these criteria identified more than $60 \%$ of pneumonia admissions defined by a more expansive criteria ${ }^{11}$ of having any of the DRG codes 79 (respiratory infections and inflammations, age $>17$ years, with complications and comorbidity), 80 (respiratory infections and inflammations, age $>17$ years, without complications and comorbidity), 89 and 90 (simple pneumonia and pleurisy, 
age $>17$ years, without complication and comorbidity). Gram-negative pneumonia, aspiration pneumonia, and pneumonias due to other specific infectious agents were not targeted in this study.

\section{Data Classifications}

The state and national cohorts were queried for the presence of adverse effects due to various antibiotics and anti-infectives, using specific ICD9 external cause of injury codes (e-codes) including e930 and e931. E-codes were developed as a supplemental code for use with the ICD and they provide a systematic way to classify diagnostic information that health care providers have entered into the medical record. E-codes have been shown to be useful and sensitive in detecting medical injuries due to drugs (including antibiotics) in hospital discharge data. ${ }^{12}$ On the basis of frequency of observed adverse drug effects (as detected by e-codes), an adverse drug effect due to an anti-infective or antibiotic was defined as that which was due to penicillins (E930.0), erythromycin and other macrolides (E930.3), tetracyclines (E930.5), cephalosporins (E930.5), sulfonamides (E931.0), quinolones (E931.3), other specified antibiotics (E930.9), other unspecified antibiotics (E931.9), or antimycobacterials (E931.8 and E930.6). Adverse drug effects due to other anti-infectives were not included due to extremely low incidence and unlikely clinical usage in pneumonia. National estimates of the number of patients experiencing an adverse drug effect were determined using discharge weights to adjust for subset sample size.

The ICD-9 codes for possible skin and allergy manifestations commonly associated with adverse drug effects were examined in patients with and without adverse drug effects as defined previously. The ICD-9 codes for skin/allergy manifestations that were considered as possibly due to adverse drug effects included erythema, not otherwise specified (695.9), flushing (782.62), Stevens-Johnson syndrome (695.1), allergic purpura (287.0), dermatitis due to drugs and medications taken internally (693.0), angioedema (995.1), unspecified allergy, (995.3), anaphylaxis not otherwise specified (NOS) (999.5), and urticaria (708). Gastrointestinal (GI) manifestations considered as possibly due to an adverse drug effect included nausea (787.02), vomiting (787.03), nausea with vomiting (787.01), diarrhea, not otherwise specified (787.91), diarrhea, other and unspecified noninfectious gas- troenteritis and colitis (558.9), or intestinal infection due to Clostridium difficile (008.45).

\section{Statistical Analysis}

Analyses were performed using JMP version 5.1 and SAS for Windows version 9 (SAS Institute, Cary, NC). In linear regression models, principal outcomes of length of stay and total hospital charges were logarithmically transformed, as this data transformation reduces the influence of outliers. ${ }^{13}$ Cases with a length of stay less than 1 day were considered to have a 23-hour LOS, to enable logarithmic transformation. Linear regression models were created to assess the impact of adverse drug effects due to antibiotics on length of stay and total charge. Linear regression models have been shown to be useful in identifying factors associated with increased hospital charges. ${ }^{13}$ Adjusting factors that were considered in multivariate models included comorbid conditions and demographic factors. Only common comorbidities that were present in greater than $5 \%$ of cases were considered and included cancer (140 through 208), congestive heart failure (428), ischemic heart disease (410-414), chronic obstructive pulmonary disease (491, 492, 496), diabetes mellitus (250), hypertension (401), asthma (493), urinary tract infection (599.0), unspecified anemia (285.9), pleural effusion (511.9), cardiac dysrhythmia (427.31), volume depletion (276.5), unspecified acquired hypothyroidism (244.9), and hypoosmolality/hyponatremia (276.1). Demographic factors such as gender, race, age, year and month of admission, and day of admission were also considered in the model. The hospital where the admission occurred was used for New York State calculations. For the national data, the region and hospital characteristics but not the hospital identification number itself were considered since not all of the same hospitals were sampled each year. Finally, the governmental health insurance status (Medicare or Medicaid for both sets of data) was considered. Medicaid and Medicaid Health Maintenance Organization (HMO) as expected reimbursement categories were considered as a single group as were Medicare and Medicare HMO in the New York State database. All of these covariants were subject to forward stepwise selection for modeling adjustment purposes. The probability required for a covariant to enter the model was 0.250 and the probability at which a covariant was removed 
from the model was 0.100 . These adjustment factors were held constant in adjusted models examining for the independent predictor effects of adverse drug reactions. To examine whether or not the presence of GI and/or skin manifestations commonly associated with adverse drug effects accounted for differences in LOS/charges, we examined whether or not adding the manifestations as a covariant would attenuate the predictor effect of the adverse drug effect.

In order to make a practical assessment of the impact of adverse drug effects on LOS and hospital charges, we chose as an example patient a 70year-old white female with a diagnosis of diabetes and hypertension, with Medicare.

Logistic regression models were used to explore comorbid conditions and demographic features that were associated with adverse drug effects within the cohort. Forward stepwise regression was used using previously described entry/ exit criteria. Odds ratios for individual predictor variables were adjusted for other significant predictor variables.

All regression models were adjusted for sampling weights in national data analyses. The time trends (year effect) for the incidences of adverse drug effects were analyzed with the GENMOD procedure in SAS, with the negative binomial distribution option. ${ }^{14,15}$

\section{RESULTS}

In the New York (SPARCS) database (NYS), 278,425 pneumonia admissions were identified. In HCUP-NIS data subsets (NIS), 186,193 pneumonia admissions formed the cohort. In both cohorts, there was a predominance of females and older patients (Table 1). Diabetes and hypertension were common comorbidities. In the NYS cohort, $1,329(0.48 \%)$ had an adverse effect related to an antibiotic or anti-infective. In the NIS cohort, an estimated $0.53 \%$ had an adverse drug effect. There was a small but significant increase in the percentage of national hospitalizations associated with an antibiotic adverse drug effect over time (time effect significance; $P=0.0149$; Table 1). However, this trend was not seen in the NYS cohort.

The most numerous adverse effects were noted in "other specified antibiotics," followed by "other unspecified antibiotics," then cephalosporins in both databases (Table 2). Cephalosporins
TABLE 1

General Characteristics of Regional and National Pneumonia Cohorts

\begin{tabular}{lll}
\hline Database & SPARCS & HCUP-NIS \\
\hline Cohort years & $2000-2005$ & $2000-2005$ \\
Cohort region & New York State & United States \\
Cohort size (identified cases) & 278,425 & 186,193 \\
Estimated actual number (n) & 278,425 & $4,547,108$ \\
$\quad$ of cases for cohort region & & \\
African American (\%) & 12.8 & 7.6 \\
Females (\%) & 53.8 & 54.4 \\
Medicare $(\%)$ & 72.9 & 72.8 \\
Mean age (years) & 72.5 & 71.4 \\
Diabetes mellitus (\%) & 25.4 & 24.4 \\
Hypertension (\%) & 41.2 & 39.0 \\
Death $(\%)$ & 6.8 & 4.7 \\
2000 ADE $(\%) / n$ & $0.44 / 205$ & $0.48 / 3372$ \\
2001 ADE $(\%) / n$ & $0.47 / 208$ & $0.53 / 3797$ \\
2002 ADE $(\%) / n$ & $0.49 / 225$ & $0.53 / 3985$ \\
2003 ADE $(\%) / n$ & $0.48 / 229$ & $0.57 / 3564$ \\
2004 ADE $(\%) / n$ & $0.52 / 249$ & $0.56 / 4250$ \\
2005 ADE $(\%) / n$ & $0.46 / 213$ & $0.60 / 4979$ \\
Total hospital charges* & $\$ 4,815,100,411$ & $\$ 70,285,286,226$ \\
\hline
\end{tabular}

NOTE: HCUP-NIS percentages, charges and age means are based on the calculated values for the entire country. $n$ refers to actual number of cases in the New York cohort and the estimated cases in the national cohort.

Abbreviation: $\mathrm{ADE}$, admissions with adverse drug effects from antibiotics as percentage of total cohort admissions.

* Charges based on actual number of cases in the New York cohort and the estimated cases in the national cohort for the entire study period.

accounted for $15 \%$ and $14 \%$ of cases with adverse drug effect due to antibiotics or anti-infectives in the NYS and NIS cohorts, respectively. Adverse drug effects due to the penicillins and quinolones were similar in frequency and were the next most common identifiable classes of antibiotics with adverse drug effects after cephalosporins. Adverse effects to other specified antibiotics and unspecified antibiotics combined constituted $59 \%$ of adverse drug effects in both NYS and NIS cohorts.

Hospitalizations associated with an adverse drug effect had higher proportions of women than hospitalizations without an adverse drug effect in both the NIS (65\% versus $54 \%$ ) and NYS (62\% versus $54 \%$ databases. Hospitalizations associated with an adverse drug effect had a mean age that was about 1 year younger than that observed in hospitalizations without an adverse drug effect in both databases. Congestive heart failure was present in a lower proportion of hospitalizations associated with an adverse drug effect compared to hospitalizations without adverse drug effects (NYS $27 \%$ versus $30 \%$, NIS $25 \%$ versus $29 \%$ ). In the NIS 
TABLE 2

Profile of Types of Adverse Drug Reactions to Different Antibiotics in the Two Cohorts

\begin{tabular}{|c|c|c|c|c|c|c|c|c|c|c|}
\hline \multirow[b]{2}{*}{ Antibiotic } & \multicolumn{5}{|c|}{ New York State (SPARCS) } & \multicolumn{5}{|c|}{ National Estimates (HCUP-NIS) } \\
\hline & $\mathrm{ADE}(\mathbf{n})$ & $\begin{array}{l}\text { ADE \% } \\
\text { of Total }\end{array}$ & $\begin{array}{l}\text { ADE with } \\
\text { Skin* (\%) }\end{array}$ & $\begin{array}{l}\text { ADE with } \\
\text { GI }^{\dagger}(\%)\end{array}$ & $\begin{array}{l}\text { ADE with GI }{ }^{\dagger} \\
\text { and/or Skin* (\%) }\end{array}$ & ADE (n) & $\begin{array}{l}\text { ADE \% } \\
\text { of Total }\end{array}$ & $\begin{array}{l}\text { ADE with } \\
\text { Skin* (\%) }\end{array}$ & $\begin{array}{l}\text { ADE with } \\
\text { GI }^{\dagger}(\%)\end{array}$ & $\begin{array}{l}\text { ADE with } \mathrm{GI}^{\dagger} \\
\text { and/or Skin* (\%) }\end{array}$ \\
\hline Penicillins & 91 & 7 & 58 & 21 & 78 & 1484 & 8 & 47 & 17 & 64 \\
\hline Erythromycin/macrolides & 102 & 8 & 28 & 44 & 71 & 1608 & 8 & 19 & 51 & 69 \\
\hline Tetracyclines & 14 & 1 & 50 & 7 & 57 & 182 & 1 & 46 & 27 & 73 \\
\hline Cephalosporins & 194 & 15 & 60 & 21 & 80 & 2684 & 14 & 55 & 19 & 69 \\
\hline Other specified antibiotics & 512 & 39 & 40 & 29 & 67 & 5986 & 30 & 37 & 29 & 62 \\
\hline Other unspecified antibiotics & 276 & 21 & 22 & 50 & 72 & 5766 & 29 & 16 & 49 & 64 \\
\hline Sulfonamide & 22 & 2 & 64 & 9 & 68 & 298 & 2 & 53 & 7 & 60 \\
\hline Quinolones & 94 & 7 & 36 & 18 & 53 & 1479 & 8 & 49 & 20 & 65 \\
\hline Antimycobacterials & 42 & 3 & 38 & 28 & 60 & 64 & 0 & 27 & 0 & 27 \\
\hline $\mathrm{ADE}$ due to any of the above & 1329 & 100 & 43 & 31 & 72 & 19740 & 100 & 34 & 33 & 65 \\
\hline
\end{tabular}

NOTE: Totals and percentages for national estimates are based on calculated values for the entire country.

*Skin/allergy manifestations of adverse drug reactions.

${ }^{\dagger}$ Gastrointestinal manifestations of adverse drug reactions.

database, adverse drug effect associated hospitalizations had a lower proportion of chronic obstructive pulmonary disease than other hospitalizations (32\% versus $40 \%$ ). Neither database showed any adverse drug effect associated disproportion with regard to hypertension and diabetes mellitus.

In logistic regression modeling, significant predictors for an adverse drug effect included non-African American race, older age, female gender, not having Medicaid, and residence outside the greater NY area (only in the NYS data). NonAfrican-Americans were more likely than AfricanAmericans to have adverse drug effect admissions (adjusted odds ratio for NYS 2.2, 95\% CI, 1.7-2.8; and for NIS 2.1, 95\% CI, 1.6-3.0). Females were more likely than males to be associated with adverse drug effect admissions (adjusted odds ratio for NYS 1.5, 95\% CI, 1.3-1.6; and for NIS 1.6, 95\% CI, 1.4-1.8). In addition, residence outside the greater NY area was associated with adverse drug effect associated admissions (adjusted odds ratio 2.1, 95\% CI, 1.8-2.3) in NYS data.

Skin and allergy manifestations potentially associated with adverse drug effects were reported in $34 \%$ and $43 \%$ of the NIS and NYS cohorts, respectively. In comparison, less than $1 \%$ of nonadverse drug effect admissions had these manifestations (Table 3) in either cohort. In NYS, adverse drug effects due to sulfonamides had a slightly higher proportion skin/allergy manifestations when compared with other antibiotic classes
TABLE 3

Proportion of Clinical Manifestations Observed in Cohort Patients with and without Antibiotic Adverse Drug Effects

\begin{tabular}{|c|c|c|c|c|}
\hline \multirow[b]{2}{*}{ Clinical Manifestation } & \multicolumn{2}{|c|}{ New York State SPARCS } & \multicolumn{2}{|c|}{$\begin{array}{c}\text { National Estimates } \\
\text { HCUP-NIS }\end{array}$} \\
\hline & $\begin{array}{l}\text { No Adverse } \\
\text { Effect }(\%)\end{array}$ & $\begin{array}{l}\text { Adverse } \\
\text { Effect }(\%)\end{array}$ & $\begin{array}{l}\text { No Adverse } \\
\text { Effect (\%) }\end{array}$ & $\begin{array}{l}\text { Adverse } \\
\text { Effect (\%) }\end{array}$ \\
\hline $\begin{array}{l}\text { Dermatitis due to drugs } \\
\text { taken internally }\end{array}$ & 0.1 & 34.1 & 0.1 & 25.9 \\
\hline Pruritus & 0.1 & 3.5 & 0.1 & 3 \\
\hline Urticaria & 0.0 & 4.0 & 0.0 & 3.6 \\
\hline Erythema & 0.0 & 0.2 & 0.0 & 0.2 \\
\hline Angioedema & 0.0 & 0.8 & 0.0 & 0.7 \\
\hline Stevens-Johnson syndrome & 0.0 & 0.3 & 0.0 & 0.2 \\
\hline Anaphylaxis & 0.0 & 0.9 & 0.0 & 0.2 \\
\hline Allergy, unspecified & 0.0 & 0.2 & 0.0 & 0.7 \\
\hline Nausea and/or vomiting & 0.6 & 6.3 & 0.9 & 7.4 \\
\hline $\begin{array}{l}\text { Diarrhea, nonspecified } \\
\text { or due to C. difficile }\end{array}$ & 3.5 & 26.5 & 3.1 & 25.5 \\
\hline
\end{tabular}

(Table 2). In contrast, NIS estimates show that adverse effects due to cephalosporins had the highest proportion of skin/allergy manifestations (Table 2). Compared to adverse drug effects due to other specified antibiotics, erythromycin/macrolides were more likely to present with GI manifestations in both databases (Table 2). Dermatitis due to drugs taken internally was coded for in $34 \%$ (NYS) and $26 \%$ (NIS) of patients that experienced an adverse drug effect, making this 
TABLE 4

Models Relating Antibiotic Adverse Effects to Hospital Charge and Length of Stay

\begin{tabular}{|c|c|c|c|}
\hline & $\begin{array}{l}\text { Example* } \\
\text { Admission } \\
\text { without ADE }\end{array}$ & $\begin{array}{l}\text { Example* } \\
\text { Admission } \\
\text { with ADE }\end{array}$ & $\begin{array}{l}P \text { Value for } \\
\text { ADE coefficient }{ }^{\dagger} \\
\text { when Added to Model }\end{array}$ \\
\hline \multicolumn{4}{|l|}{ SPARCS } \\
\hline \multicolumn{4}{|l|}{ Hospital charge } \\
\hline Simple unadjusted model & $\$ 12,274$ & $\$ 13,045$ & 0.007 \\
\hline Adjusted model for comorbidities and demographics & $\$ 14,160$ & $\$ 17,533$ & $<0.0001$ \\
\hline Adjusted model for comorbidities and demographics including $\mathrm{GI}^{\ddagger}$ manifestations & $\$ 18,865$ & $\$ 21,560$ & $<0.0001$ \\
\hline Adjusted model for comorbidities and demographics including skin/allergy ${ }^{\S}$ manifestations & $\$ 16,777$ & $\$ 19,370$ & $<0.0001$ \\
\hline Adjusted model for comorbidities and demographics including $\mathrm{GI}^{\dagger}$ and skin/allergy ${ }^{\S}$ manifestations & $\$ 23,227$ & $\$ 24,275$ & 0.0227 \\
\hline \multicolumn{4}{|l|}{ LOS (days) } \\
\hline Simple unadjusted model & 5.4 & 6.7 & $<0.0001$ \\
\hline Adjusted model for comorbidities and demographics & 4.0 & 5.2 & $<0.0001$ \\
\hline Adjusted model for comorbidities and demographics including $\mathrm{GI}^{\dagger}$ manifestations & 5.4 & 6.5 & $<0.0001$ \\
\hline Adjusted model for comorbidities and demographics including skin/allergy ${ }^{\S}$ manifestations & 4.8 & 5.8 & $<0.0001$ \\
\hline Adjusted model for comorbidities and demographics including $\mathrm{GI}^{\dagger}$ and skin/allergy ${ }^{\S}$ manifestations & 6.8 & 7.4 & $<0.0001$ \\
\hline \multicolumn{4}{|l|}{ HCUP } \\
\hline \multicolumn{4}{|l|}{ Hospital charge } \\
\hline Simple unadjusted model & $\$ 10,840$ & $\$ 11,917$ & 0.0008 \\
\hline Adjusted model for comorbidities and demographics & $\$ 12,941$ & $\$ 14,284$ & $<0.0001$ \\
\hline Adjusted model for comorbidities and demographics including $\mathrm{GI}^{\ddagger}$ manifestations & $\$ 14,923$ & $\$ 15,781$ & 0.0208 \\
\hline Adjusted model for comorbidities and demographics including skin/allergy ${ }^{\S}$ manifestations & $\$ 13,543$ & $\$ 14,723$ & 0.0092 \\
\hline Adjusted model for comorbidities and demographics including $\mathrm{Gl}^{\dagger}$ and skin/allergy ${ }^{\S}$ manifestations & $\$ 15,887$ & $\$ 16,453$ & 0.1784 \\
\hline \multicolumn{4}{|l|}{ LOS (days) } \\
\hline Simple unadjusted model & 4.4 & 5.1 & $<0.0001$ \\
\hline Adjusted model for comorbidities and demographics & 4.0 & 4.9 & $<0.0001$ \\
\hline Adjusted model for comorbidities and demographics including $\mathrm{GI}^{\dagger}$ manifestations & 4.8 & 5.5 & $<0.0001$ \\
\hline Adjusted model for comorbidities and demographics including skin/allergy ${ }^{\S}$ manifestations & 4.0 & 4.7 & $<0.0001$ \\
\hline Adjusted model for comorbidities and demographics including $\mathrm{GI}^{+}$and skin/allergy ${ }^{\S}$ manifestations & 5.3 & 5.9 & $<0.0001$ \\
\hline
\end{tabular}

NOTE: NYS LOS and charge models had adjusting comorbid demographic factors, which included race, age, gender, hospital, year, month, Medicare, Medicaid, congestive heart failure, diabetes mellitus, hypertension, cancer, ischemic heart disease, volume depletion cardiac arrhythmia, urinary tract infection, pleural effusion, unspecified anemia, hypothyroidism, and hyponatremia. National LOS and charge models had adjusting comorbid demographic factors, which included race, age, gender, hospital size, census region, teaching hospital status, hospital ownership class, rural location, year, month, day, Medicare, Medicaid, congestive heart failure, diabetes mellitus, hypertension, cancer, ischemic heart disease, volume depletion cardiac arrhythmia, urinary tract infection, pleural effusion, unspecified anemia, hypothyroidism (not in LOS model), asthma, and hyponatremia.

Abbreviation: ADE, adverse drug effect.

*NYS example admission: 70-year-old white female with diabetes mellitus, hypertension, and Medicare admitted in December 2004 at St. Vincent's Hospital, Staten Island. NIS example admission: 70-year-old white female with diabetes mellitus, hypertension, and Medicare admitted in June 2004 in the Western region at a large teaching nonrural hospital on a non-weekend day.

${ }^{\dagger} P$ value for analysis of variance (ANOVA) F-test testing for the null hypothesis that the ADE factor's coefficient $=0$, main effects general linear model.

\$ GI manifestations include nausea, vomiting, or diarrhea (unspecified or due to C. difficile).

${ }^{\S}$ Skin/allergy manifestations include pruritus, anaphylaxis, angioedema, erythema, allergy NOS, urticaria, Stevens-Johnson syndrome, and dermatitis due to medication taken internally.

condition the most common skin/allergy manifestation associated with an adverse drug effect (Table 3). This was followed in frequency by urticaria and pruritus. Diarrhea was also a common symptom related to adverse drug effects (Table 3). While $72 \%$ of adverse drug effects had either GI or skin/allergy manifestations in the NYS cohort, only $65 \%$ of the NIS cohort had these manifestations reported. No increase in mortality was observed in patients with adverse drug effects compared to those without adverse drug effects (data not shown).
Both databases showed that adverse drug effects affected both LOS and total charges (Table 4). In the NIS database, adjusted models showed that GI manifestations impacted hospital charges more than skin/allergy manifestations (Table 4). In both the NYS and NIS cohorts, the effect of adverse drug effects on hospital charges was attenuated after accounting for skin/allergy and GI manifestations. However, even after accounting for both manifestations, there still was a significant adverse drug effect influence on LOS. In the example patient, predicted excess hospitalization 
charges associated with the presence of an adverse drug effect was $\$ 1,243$ and $\$ 3,373$ for the NIS and NYS cohorts, while LOS increases associated with an adverse drug effect were about 1 day in both cohorts. Linear regression models, which included adjustment factors including comorbidities and demographic/financial factors, showed that the models accounted for $13 \%$ of the variance $\left(\mathrm{R}^{2}\right.$ values) in LOS and $40 \%$ in charges for the NYS but only $7 \%$ for LOS and 15\% for charges for the NIS.

\section{DISCUSSION}

This study documents antibiotic related adverse drug effects as a predictable, but infrequent complication that occurs in adult patients hospitalized for pneumonia. While the incidence of antibioticrelated adverse drug events has been calculated in both hospital ${ }^{2}$ and nursing home ${ }^{16}$ populations, these studies have not specified admissions that were associated with antibiotic use. Thus calculations of antibiotic adverse drug events for actual at-risk patients (ie, those receiving antibiotics) are imprecise in these reports. In the present study, it is highly probable that nearly all admissions were associated with actual antibiotic administration. Thus, a rough incidence of an identifiable antibiotic adverse drug effect for adult pneumonia admissions can be expected to be roughly $0.5 \%$. Of interest is the observation that the national incidence of this complication appears to be increasing slightly but steadily in recent years. This could be explained on the basis of increased coding slots or DRG creep ${ }^{17}$ in more recent years with the national cohort. Layde et al. ${ }^{12}$ utilized ecodes to identify medical injury due to medications in Wisconsin hospital discharge data (excluding newborn delivery discharges), unselected for infectious diseases. They calculated an overall $0.5 \%$ incidence of antibiotic-associated adverse effects. Since not all hospitalizations are associated with antibiotic administration, this would imply that the incidence of antibiotic associated adverse effects in hospitalized patients actually given antibiotics would be higher than $0.5 \%$. The relatively low incidence of antibioticassociated adverse effects observed in the present study may relate to briefer and less complex hospitalizations for these patients compared with other patients treated with antibiotics. The use of ICD-9 codes (including e-codes) as flags of adverse drug events may also underestimate actual rates. In a study of Utah hospitalization discharges in 2001,
Hougland et al. ${ }^{18}$ found that these flags had a sensitivity of $55 \%$ for confirmed adverse drug events of various categories. This contrasts with the $98.5 \%$ sensitivity of e-codes to identify medical injury due to drugs, described in the Wisconsin hospital discharge data. The distinction between adverse drug effects, adverse drug reactions, and adverse drug events may be important in understanding these data. Adverse drug reactions have been defined as "any noxious, unintended, and undesired effect of a drug, which occurs at doses used in humans for prophylaxis, diagnosis, or therapy" and can be considered a subset of adverse drug events, which, unlike adverse drug reactions, may also be due to drug administration errors. ${ }^{6}$ The term "adverse drug effect" has been used more in pharmacology literature $^{4}$ and in medical coding, ${ }^{18}$ and may refer more to known side effects of medications, whereas adverse drug events have been broadly defined as "an injury resulting from administration of a drug." 6 As this study utilized medical coding for data abstraction, we used the term "adverse drug effect" for all results.

Determining factors that influence hospital charges and length of stay are complex. Regional differences ${ }^{19}$ may be 1 factor, as suggested by difference in charges for the example admission models (Table 4). The multivariate analyses showed that nearly $40 \%$ of the variation in total New York State hospitalization charges could be explained on the basis of demographic, comorbidity factors, and between hospital variation, with an additional independent effect identifiable in the presence of an adverse drug effect to an antibiotic. The explained variance in the New York State hospitalization charges exceeds that observed in other published clinical predictor models of hospital charges based on statewide or province-wide discharge databases. ${ }^{12,20,21}$ Although less variability was explained in national models, independent adverse drug effects influence on both total charge and length of stay were also observed. Higher charges with adverse drug effect associated admissions could be explained in part by increased illness severity, leading to more hospital days, and thus higher charges in per diem reimbursement schemes. In DRG-based reimbursements, adverse drug effect presence in an admission, with its attendant increased length of stay, could be considered an outlier case, in which case an increased inpatient prospective payment system (IPPS) payment would be authorized in addition to the base payment. ${ }^{22}$ 
Because the impact of adverse drug effects on length of stay was still present even after controlling for both skin/allergy and GI manifestations, this may suggest that other factors relating to the adverse drug effects may have influenced LOS. These factors might include physician reluctance to discharge these patients or the influence of other organ systems not accounted for or reported in this study. The attenuation of the adverse drug effect's influence on hospital charge models by including possible clinical manifestations of adverse drug effects suggests that these manifestations were the main contributors to higher charges associated with adverse drug effect admissions. These disparate findings regarding adverse drug effects on LOS versus charges are consistent with the notion that hospital LOS and hospital charge are separate constructs, which may be associated with separate factors affecting these outcomes. ${ }^{23}$ The $13 \%$ variation in LOS explained in the New York state hospitalization regression models was similar to the $14 \%$ variation in LOS observed in clinical predictor models for congestive heart failure hospitalizations in New York state. ${ }^{24}$ Layde et al. ${ }^{12}$ found a $14.5 \%$ and $18.5 \%$ adjusted increase in charges and LOS associated with any medical injury in Wisconsin hospital discharges. The excess length of stay due to antibiotic related medical injury was 1.27 days. This magnitude of effect is comparable to that observed in the present study.

There are a number of limitations in this study related to coding practices and the retrospective nature of the investigation. Currently, there are no ICD-9 e-codes for adverse effects due to commonly used older antibiotics such as vancomycin, clindamycin, and metronidazole, or to the newer antibiotic classes. Since currently recommended treatments for community-acquired pneumonia ${ }^{25}$ are among the specified drugs with adverse effect coding, and since other specified and unspecified anti-infective drug-associated (no drug names or categories provided) adverse effects were frequent, it suggests that infectious processes other than community-acquired pneumonia were also being treated in many hospitalizations. Another limitation is that because the temporal sequence of events cannot be ascertained with this data, it is possible that an adverse drug effect was due to an antibiotic given prior to hospitalization. It has been suggested that "onset of diagnosis" or "present on admission" information be part of a new administrative data coding strategy which has been used in some states in the US. ${ }^{19}$ The adoption of ICD-10 codes may allow for more specificity and detailing of adverse drug reactions using administrative data, as described for the United Kingdom by Waller et al. $^{26}$ The actual incidence of hospitalization-associated adverse drug effects could be underestimated if a significant number of adverse effects occurred after discharge as only in-hospital events were recorded.

In summary, we found that although the incidence of adverse drug effects is small, there is a definite quantifiable impact of these adverse effects on LOS and hospital charges in patients hospitalized with pneumonia. To our knowledge, there have not been similar large-scale database studies to evaluate the incidence and impact of adverse drug effects related to antibiotics in both national and statewide samples. These findings also have implications in studies of outcomes related to pneumonia hospitalizations.

Address for correspondence: Robert Y. Lin, MD, St Vincents Hospital-Manhattan-SVCMC, 153 West 11th Street, New York, NY 10011; Telephone: 212604-8460; Fax: 212-604-3134; E-mail: robert_lin@nymc.edu

The authors thank Ariel D. Teitel, MD, for his assistance. This study was supported in part by the intramural scholarship program at New York Medical College.

Received 24 October 2007; revision received 25 June 2008; accepted 13 July 2008.

\section{REFERENCES}

1. Bates DW, Cullen DJ, Laird N, et al. Incidence of adverse drug events and potential adverse drug events: implications for prevention. JAMA. 1995;274:29-34.

2. Classen DC, Pestotnik SL, Evans RS, et al. Adverse drug events in hospitalized patients. JAMA. 1997;277:301-306.

3. Lazarou J, Pomeranz BH, Corey PN. Incidence of adverse drug reactions in hospitalized patients. JAMA. 1998;279: 1200-1205.

4. Beringer PM, Wong-Beringer A, Rho JP. Economic aspects of antibacterial adverse effects. Pharmacoeconomics. 1998;13:35-49.

5. Popovic JR, Hall MJ. 1999 National Hospital Discharge Survey. Advance data from vital and health statistics; no 319. Hyattsville, MD: National Center for Health Statistics; 2001.

6. Lazarou J, Pomeranz BH, Corey PN. Incidence of adverse drug reactions in hospitalized patients: a meta-analysis of prospective studies. JAMA. 1998;279:1200-1205.

7. Lin RY, Pitt MD, Lou WYW, Yi Q. Asthma hospitalization patterns in young children relating to admission age, infection presence, sex, and race. Ann Allergy Asthma Immunol. 2007;98:139-145.

8. Lin RY, Cannon AG, Teitel AD. Pattern of hospitalizations for angioedema in New York between 1990 and 2003. Ann Allergy Asthma Immunol. 2005;95:159-166.

9. Inpatient Output Data Dictionary, SPARCS. Available at: http://www.health.state.ny.us/statistics/sparcs/inpat.htm. Accessed October 2008. 
10. Agency for Healthcare Research and Quality. The HCUP Nationwide Inpatient Sample (NIS). Rockville, MD: Agency for Healthcare Research and Quality; 2004.

11. Aronsky D, Haug PJ, Lagor C, Dean NC. Accuracy of administrative data for identifying patients with pneumonia. Am J Med Qual. 2005;20:319-328.

12. Layde PM, Meurer LM, Guse C, et al. Medical injury identification using hospital discharge data. Advances in Patient Safety. Vol 2. Rockville, MD: Agency for Healthcare Research and Quality; 2007. Available at: http://www.ahrq.gov/downloads/ pub/advances/vol2/Layde.pdf. Accessed October 2008.

13. Austin PC, Ghali WA, Tu JV. A comparison of several regression models for analyzing cost of CABG surgery. Stat Med. 2003;22:2799-2815.

14. University of Kentucky. Poisson and negative binomial regressions. Available at: http://www.uky.edu/ComputingCenter/SSTARS. Accessed October 2008.

15. Gardner W, Mulve EP, Shaw EC. Regression analyses of counts and rates: Poisson, overdispersed Poisson, and negative binomial models. Psychol Bull. 1995;118;392-404.

16. Gurwitz JH, Field TS, Avorn J, et al. Incidence and preventability of adverse drug events in nursing homes. Am J Med. 2000;1;109:87-94.

17. Zhan C, Miller MR. Administrative data based patient safety research: a critical review. Qual Saf Health Care. 2003;12(Suppl 2):ii58-ii63.

18. Hougland P, Xu W, Pickard S, et al. Performance of International Classification of Diseases, 9th Revision, Clinical Modification codes as an adverse drug event surveillance system. Med Care. 2006;44:629-636.
19. Pelletier AJ, Mansbach JN, Camargo CA. Direct medical costs of bronchiolitis hospitalizations in the United States. Pediatrics Dec. 2006;118:2418-2423.

20. Shahian DM, Heatley GJ, Westcott GA. Relationship of hospital size, case volume, and cost for coronary artery bypass surgery: analysis of 12,774 patients operated on in Massachusetts during fiscal years 1995 and 1996. J Thorac Cardiovasc Surg. 2001;122:53-64.

21. Elixhauser A, Steiner C, Harris DR, Coffey RM. Comorbidity measures for use with administrative data. Med Care. 1998;36:8-27.

22. Health and Human Services. Centers for Medicaid and Medicare Services (CMS) Home. Medicare. Acute Inpatient PPS. Overview. Available at: http://www.cms.hhs.gov/Acute InpatientPPS. Accessed October 2008.

23. Ghali WA, Hall RE, Ash AS, Moskowitz MA. Identifying preand postoperative predictors of cost and length of stay for coronary artery bypass surgery. Am J Med Qual. 1999:14: 248-254.

24. Polanczyk CA, Lane A, Coburn M, et al. Hospital outcomes in major teaching, minor teaching, and nonteaching hospitals in New York state. Am J Med. 2002;112:255-261.

25. Mandell LA, Wunderink RG, Anzueto A, et al. Infectious Disease Society/American Thoracic Society Consensus Guidelines on the Management of Community-Acquired Pneumonia in Adults. Clin Infect Dis. 2007;44:S27-S72.

26. Waller P, Shaw M, Ho D, Shakir S, Ebrahim S. Hospital admissions for 'drug-induced' disorders in England: a study using the Hospital Episodes Statistics (HES) database. $\mathrm{Br} \mathrm{J}$ Clin Pharmacol. 2005;59:213-219. 